\title{
JAK2V617F allele burden is associated with thrombotic mechanisms activation in polycythemia vera and essential thrombocythemia patients
}

\author{
Margarida Coucelo • Gonçalo Caetano • Teresa Sevivas • Susana Almeida Santos • \\ Teresa Fidalgo • Celeste Bento • Manuela Fortuna • Marta Duarte • \\ Cristina Menezes • M. Letícia Ribeiro
}

Received: 20 August 2013/Revised: 11 November 2013/Accepted: 13 November 2013/Published online: 26 November 2013

(c) The Japanese Society of Hematology 2013

\begin{abstract}
The clinical courses of polycythemia vera (PV) and essential thrombocythemia (ET) are characterized by thrombohemorrhagic diathesis. Several groups have suggested an association between JAK2V617F mutation and thrombosis. We hypothesized a relationship between JAK2V617F allele burden, cellular activation parameters, and thrombosis. We evaluated a group of PV and ET patients using flow cytometry: platelet CD62P, CD63, and dense granules, platelet-leukocyte aggregates (PLA), leukocyte CD11b and monocyte tissue factor (TF) expression. All patients had increased baseline platelet CD62P and CD63 expression $(p<0.05) ; 71 \%$ of PV and $47 \%$ of ET presented with a storage pool disease. Leukocyte CD11b, $\mathrm{TF}$, and PLA were elevated in all patients. TF was higher in PV compared to ET $(p<0.05)$ and platelet-neutrophil [polymorphonuclear (PMN)] aggregates were increased in ET versus PV $(p<0.05)$. In ET, PLA were correlated with platelet numbers $(p<0.05)$. In all patients, JAK2V617F allele burden was directly correlated with monocyte CD11b. Patients with JAK2V617F allele burden $>50 \%$ presented higher levels of leukocyte activation. In ET, thrombosis was associated with JAK2V617F mutation $\left(p<0.05, \quad \chi^{2}=5.2\right), \quad$ increased monocyte CD11b $(p<0.05)$ and with platelet-PMN aggregates $(p<0.05)$. In ET patients, hydroxyurea does not significantly reduce the activation parameters. Our data demonstrate that JAK2V617F allele burden is directly correlated with
\end{abstract}

M. Coucelo $(\varangle)$ - G. Caetano · T. Sevivas · S. Almeida Santos . T. Fidalgo $\cdot$ C. Bento $\cdot$ M. Fortuna $\cdot$ M. Duarte $\cdot$ C. Menezes $~$ M. L. Ribeiro

Serviço Hematologia, Centro Hospitalar e Universitário de Coimbra, Coimbra, Portugal, Av. Afonso Romão, Santo António dos Olivais, 3000-075 Coimbra, Portugal

e-mail: margarida.coucelo@chc.min-saude.pt activation parameters that drive mechanisms that favor thrombosis.

Keywords JAK2V617F - Myeloproliferative neoplasms $\cdot$ Platelet activation · Leukocyte activation

\section{Introduction}

Polycythemia vera (PV) and essential thrombocythemia (ET) are the commonest Philadelphia-negative myeloproliferative neoplasms (MPN). The clinical course of these diseases is characterized by microcirculatory disturbances and increased risk of both arterial and venous thromboses, which often manifest at diagnosis or in the preclinical phase [1]. Bleeding manifestations, although less common, are typically mucocutaneous, suggesting a defective primary hemostasis and may be concomitant with thrombotic complications $[1,2]$. Concerning to the hemorrhagic pattern a number of platelets defects have been identified, including acquired storage pool disease, platelet membrane abnormalities, and abnormal arachidonic acid metabolism [2-4]. In the pathogenesis of thrombosis, several factors are likely to be involved, including leukocyte number, platelets, and leukocytes activation and interaction, microparticles formation and endothelial damage [2, 4-6]. Recent investigations demonstrated that polymorphonuclear (PMN) leukocytes of both PV and ET patients present phenotypical changes of an activation status, such as increased expression of the membrane $\beta 2$ integrin $\mathrm{CD} 11 \mathrm{~b}$ and leukocyte alkaline phosphatase, and a significant increase in the cellular content of elastase [7-9]. In vitro, interactions between platelets, neutrophils and monocytes promotes the generation of neutrophil/platelets aggregates and increases procoagulant activity of monocytes by 
inducing tissue factor (TF) expression [7-10]. After the discovery of JAK2V617F mutation, different groups found a correlation between the presence of JAK2 $2617 \mathrm{~F}$ mutation and increased activation patterns, suggesting that this mutation may be associated with increased activation of leukocytes and platelets in MPN [11, 12]. More recently, several evidences support a relationship between JAK2V617F allele burden and disease phenotype [11-14]. Also, JAK2V617F mutation and allele burden have been related with thrombosis both in ET and PV [13, 14]. The aim of this study was to characterize platelet and leukocyte activation status in a group of PV and ET patients and investigate the influence of JAK2V617F allele burden in specific haemostatic abnormalities and thrombo-hemorrhagic features.

\section{Materials and methods}

\section{Subjects}

After informed consent, $31 \mathrm{PV}$ and 49 ET patients, diagnosed according to the WHO 2008 classification, were enrolled in this study. All PV and 32/49 ET patients have the JAK2V617F mutation. All PV and 41 ET patients are under treatment with hydroxyurea (HU); 1 patient with ET was on busulfan and 7 ET were not receiving any specific treatment. With patients' informed written consent, aspirin was withdrawn for 10 days prior flow cytometry studies. Twelve patients with PV (39\%) and 16 with ET (33\%) suffered thrombotic events; 7 PV (23\%) and 10 ET (20\%) had minor hemorrhage. Vascular events included ischemic stroke, cerebral transient ischemic attack, deep vein thrombosis. Minor hemorrhage included epistaxis and gingival hemorrhage. Forty-eight healthy subjects with no thrombohemorrhagic history acted as control group. Patients and controls data are summarized in Table 1.

Blood samples and reagents

Peripheral blood samples were obtained in trisodium citrate tubes (vacutainer system) and the first $3 \mathrm{ml}$ of blood were discarded. Platelet studies were initiated within $1 \mathrm{~h}$ after blood collection and leukocyte studies were accomplished within a maximum of $4 \mathrm{~h}$ after sample collection. All the monoclonal antibodies were purchased from BectonDickinson (BD, USA). Platelets were activated using Thrombin Receptor Activator Peptide 6 (TRAP6) and arachidonic acid sodium salt (Sigma Chemical, St Louis, USA). Quinacrine (Sigma Chemical, UK) was used to detect platelets dense granules.

\section{Molecular studies}

DNA was extracted from whole blood using standard procedures. JAK2V617F mutation was detected by allelespecific polymerase chain reaction (ASO-PCR) [15]. JAK2V617F allele burden quantification was performed in genomic DNA using JAK2MutaQuant ${ }^{\mathrm{TM}}$ Kit (Ipsogen) according to manufacture instructions (Real-Time PCR 7300, Applied Biosystems). In ET, patients were excluded

Table 1 Characteristics of patients and controls

\begin{tabular}{|c|c|c|c|c|c|}
\hline & \multirow{2}{*}{$\begin{array}{l}\text { PV } \\
\text { JAK2 }{ }^{\mathrm{V} 617 \mathrm{~F}} \text { mutated pts }\end{array}$} & \multicolumn{3}{|l|}{ ET } & \multirow[t]{2}{*}{ Controls } \\
\hline & & All patients & JAK2 $2^{\mathrm{V} 617 \mathrm{~F}}$ wild-type pts & $\mathrm{JAK} 2^{\mathrm{V} 617 \mathrm{~F}}$ mutated pts & \\
\hline Subjects & 31 & 49 & 17 & 32 & 48 \\
\hline Male/female & $13 / 18$ & $22 / 27$ & $7 / 10$ & $15 / 17$ & $15 / 33$ \\
\hline JAK2 allele burden (\%) & $56.6 \pm 30^{\phi}$ & - & - & $35.4 \pm 22.5$ & - \\
\hline Age (years) & $73.7 \pm 10.7$ & $65.8 \pm 13.3$ & $58 \pm 14$ & $70.2 \pm 10.5$ & $33.8 \pm 12.1$ \\
\hline Hemoglobin (g/dL) & $13.9 \pm 1.6$ & $13.8 \pm 1.4$ & $13 \pm 1$ & $14.1 \pm 1.4$ & $14.2 \pm 1.3$ \\
\hline WBC $\left(10^{9} / \mathrm{L}\right)$ & $6.9 \pm 2.9$ & $6.9 \pm 2.1$ & $7 \pm 2$ & $7.0 \pm 2.2$ & $7.1 \pm 1.7$ \\
\hline Platelets $\left(10^{9} / \mathrm{L}\right)$ & $292 \pm 148$ & $480 \pm 149 *$ & $527 \pm 178$ & $455 \pm 127$ & $288 \pm 74$ \\
\hline Follow-up (years) & $8.4 \pm 5.7$ & $7.8 \pm 5.6$ & $8 \pm 5$ & $7.8 \pm 6.1$ & - \\
\hline Thrombosis & 12 & 16 & 2 & 14 & - \\
\hline Arterial (\%) & $9(75)$ & $15(94)$ & $2(100)$ & $13(93)$ & - \\
\hline Venous (\%) & $3(25)$ & $1(6)$ & - & $1(7)$ & - \\
\hline Hemorrhage & 7 & 10 & 0 & 10 & - \\
\hline
\end{tabular}

Values expressed as mean $\pm \mathrm{SD}$

ф $p<0.006$ versus ET

$* p<0.05$ versus $\mathrm{PV}$ and $\mathrm{CT}$ 
mutations in MPL exon 10 by sequencing (ABI 310 Genetic Analyzer, Applied Biosystems).

Flow cytometry

\section{Platelet baseline and activation studies}

A whole blood flow cytometry assay was used to evaluate platelet P-selectin (CD62P) and CD63 (dense granules). For activation assays, AA and TRAP6 were added at final concentration of $0.1 \mathrm{mM}, 5$, and $25 \mu \mathrm{M}$, respectively. Ten thousand events were collected and platelets were identified by their characteristic side-scatter and FITC-conjugated anti-CD42b positivity. Results for CD62P and CD63 were expressed as percentage.

\section{Platelet mepacrine uptake and release test}

Citrated whole blood was diluted and incubated with mepacrine $40 \mu \mathrm{M}$ (uptake test); for release test TRAP6 $30 \mu \mathrm{M}$ was added to the tube. Samples were resuspended and analyzed in the FACSCalibur (BD). Ten thousand events were collected and platelets were identified by their forward/side-scatter logarithmic mode, and green fluorescence (FL1) associated to mepacrine was quantitated. A positive result was recorded when mean fluorescence intensity (MFI) ratio between mepacrine uptake and release was $>1.5$. For each analysis, a normal control sample was included.

\section{Evaluation of platelet-leukocyte aggregates}

Whole blood samples were incubated with FITC-conjugated anti-CD42b, PE-conjugated anti-CD14, and PerCPconjugated anti-CD45. After incubation, samples were lysed, centrifuged, resuspended in PBS, and analyzed on the FACSCalibur (BD). 50,000 events were collected for each sample. Aggregates of platelet-PMN leukocytes were identified by forward/side-scatter PMN proprieties and CD42b positivity and platelet-monocyte (PM) aggregates were identified by CD14 and CD42b positivity. PMN and monocyte aggregates were expressed as percentages.

\section{CD11b and tissue factor (CD142) expression in PMN and monocytes}

Whole blood samples were incubated with FITC-conjugated anti-CD14, PE-conjugated anti-CD11b, or anti-CD142 PE and PerCP-conjugated anti-CD45. After incubation at room temperature, samples were lysed, centrifuged, and resuspended in PBS, before analysis in the FACSCalibur (BD). PMN and monocytes were identified by their forward and side-scatter properties and monocytes were identified by gating the CD14-positive cells; 50,000 events were collected from each sample. CD11b is constitutively expressed in PMN and monocytes, and the number increases upon activation, therefore the results are expressed as MFI units; $\mathrm{CD} 142$, TF, is a glycoprotein synthesized by monocytes, and because is not constitutively expressed the results are given as percentage of positive cells. To circumvent the day-to-day variations standardized fluorescent beads (Quantum ${ }^{\mathrm{TM}}$ PE Medium Level, BangsLabs, USA) were used to converted MFI values in molecules of equivalent soluble fluorochrome (MESF).

Statistical analysis

All results are expressed as mean $\pm \mathrm{SD}$. The difference analysis was performed using the Student's $t$ test for paired data. For multiple comparison analysis one-way ANOVA test followed by Tukey post hoc analysis was used. Chi squared $\left(\chi^{2}\right)$ was used to determine significance between nominal variables. The nonparametric Spearman rank correlation was used to test for correlation between JAK2V617F allele burden and ordinal variables. StatView 5 and GrapPad Prism were used for statistical analysis. Differences were considered significant at a $p$ value $<0.05$.

\section{Results}

Platelet activation studies

We evaluated platelet baseline expression of alpha (CD62P) and dense granules (CD63) as markers of in vivo activation. To assess platelets degranulation, we submitted the platelets to stimulus with different agonists. Results are summarized in Table 2. Baseline platelets CD62P and CD63 expression were significantly elevated in patients with MPN compared with controls $(p<0.0001)$. Platelets response to arachidonic acid was significantly increased in both PV and ET $(p<0.0001)$. Regarding TRAP6 agonist, no difference was found in CD62P expression after exposure to low TRAP6 $(5 \mu \mathrm{M})$ concentration, however, statistically significant differences were observed after exposure to a higher concentration of TRAP6 $(25 \mu \mathrm{M})$ $(p=0.0003)$. CD63 expression was significantly diminished after TRAP6 activation, comparing to controls $(p<0.0001)$.

Mepacrine uptake and release test

Platelets mepacrine uptake and release tests were abnormal (ratio <1.5) in $71 \%$ of PV and $47 \%$ of ET patients. In all the controls the test was positive. A statistically significant 
Table 2 Platelet activation studies and mepacrine uptake and release test

\begin{tabular}{|c|c|c|c|c|}
\hline & $\begin{array}{l}\mathrm{PV} \\
(n=31)\end{array}$ & $\begin{array}{l}\mathrm{ET} \\
(n=49)\end{array}$ & $\begin{array}{l}\text { Controls } \\
(n=48)\end{array}$ & $p$ value* \\
\hline \multicolumn{5}{|l|}{ CD62P (\%) } \\
\hline Baseline & $8.0 \pm 3.7$ & $10.4 \pm 10.3$ & $3.1 \pm 1.4$ & $<0.0001^{\circ}$ \\
\hline $\begin{array}{l}\text { AA } \\
0.1 \mathrm{mM}\end{array}$ & $29.1 \pm 9.6$ & $27.4 \pm 17.3$ & $13.7 \pm 8.1$ & $<0.0001^{\circ}$ \\
\hline $\begin{array}{l}\text { TRAP6 } \\
5 \mu \mathrm{M}\end{array}$ & $57.2 \pm 21.2$ & $61.7 \pm 27.0$ & $71.2 \pm 28.5$ & NS \\
\hline $\begin{array}{l}\text { TRAP6 } \\
25 \mu \mathrm{M}\end{array}$ & $83.8 \pm 16.1$ & $86.7 \pm 16.9$ & $95.9 \pm 3.3$ & $0.0003^{\circ}$ \\
\hline \multicolumn{5}{|l|}{ CD63 (\%) } \\
\hline Baseline & $5.2 \pm 4.4$ & $4.3 \pm 5.3$ & $2.0 \pm 0.8$ & $0.001^{\circ}$ \\
\hline $\begin{array}{l}\text { TRAP6 } \\
5 \mu \mathrm{M}\end{array}$ & $22.1 \pm 14.3$ & $26.1 \pm 18.4$ & $14.7 \pm 16.1$ & $0.0038^{\circ}$ \\
\hline $\begin{array}{l}\text { TRAP6 } \\
25 \mu \mathrm{M}\end{array}$ & $48.5 \pm 23$ & $51.9 \pm 18.9$ & $75.3 \pm 13.3$ & $<0.0001^{\circ}$ \\
\hline $\begin{array}{l}\text { Mepacrine } \\
\text { uptake } \\
(\mathrm{MFI})\end{array}$ & $9.2 \pm 3.6$ & $9.0 \pm 2.4$ & $11.3 \pm 2.0$ & $<0.0001^{\circ}$ \\
\hline $\begin{array}{l}\text { Mepacrine } \\
\text { release } \\
(\mathrm{MFI})\end{array}$ & $6.6 \pm 2.9$ & $6.0 \pm 2.3$ & $5.6 \pm 1.5$ & NS \\
\hline $\begin{array}{l}\text { Mepacrine } \\
\text { test normal }\end{array}$ & $8(n=28)$ & $25(n=47)$ & $46(n=46)$ & - \\
\hline
\end{tabular}

Values expressed as mean $\pm \mathrm{SD}$

NS not significant, MFI mean fluorescence intensity

* One-way ANOVA test followed by post hoc analysis using Tukey multiple comparison test

Significant versus control group

reduced mepacrine uptake was observed in PV and ET patients (Table 2).

Circulating platelet-monocyte and platelet-neutrophil aggregates

The results show a statistically significant increased percentage of PM and PMN-aggregates in both ET and PV patients compared with the control group (Fig. 1). Particularly, the median percentage of PM-aggregates was $86.6 \%$ in ET and $80.2 \%$ in PV versus $68 \%$ in controls $(p<0.0001)$; and the median percentage of PMN-aggregates was $27.3 \%$ in ET patients $(p<0.0001)$ and $21.6 \%$ in PV patients $(p<0.01)$ versus $15.2 \%$ in controls. ET patients present a statistically significant increase in PMNaggregates comparing to $\mathrm{PV}$ patients $(p=0.015)$.

Monocyte and neutrophil CD11b expression and monocyte tissue factor expression

In both group of patients, monocyte-CD11b was statistically significant increased (PV: $p=0.001$; ET: $p=0.001$ ) compared to the control group (Fig. 1). PMN-CD11b, in both group of patients had no statistically significant differences comparing to controls. Monocyte TF (CD142) expression was statistically significant increased in $\mathrm{PV}$ versus ET patients $(p=0.0028)$ and controls $(p<0.0001)$; although higher in ET patients than in controls, no significant differences were found (Fig. 1).

Correlation analyses

In PV patients, we found a statistically significant correlation between JAK2V617F allele burden and PMNaggregates- $(p=0.002, \quad r=0.6), \quad \mathrm{CD} 11 \mathrm{~b}$ expression (monocytes: $p=0.001, r=0.6$; neutrophils: $p=0.05$, $r=0.4)$, and monocyte TF expression $(p<0.0001$, $r=0.7)$. In ET group, monocyte CD11b expression was directly correlated with JAK2V617F allele burden $(p=0.003, r=0.5)$ and platelet-leukocyte aggregates (PLA) were correlated with platelet numbers (PM: $p=0.02, r=0.4$; PMN: $p=0.01, r=0.4)$. Monocyte CD11b expression and monocyte TF expression were positively correlated (PV: $p<0.0011, r=0.6$; ET: $p=0.02, r=0.4)$. In both groups of patients, we found an inverse correlation between JAK2V617F allele burden and CD63 expression after TRAP6 $(25 \mu \mathrm{M})$ (PV: $p=0.004$, $r=-0.5 \mathrm{ET}: p=0.002, r=-0.5)$. To evaluate difference, the influence of JAK2V617F allele burden, patients were divided into groups. PV patients were divided into two groups: JAK2V617F allele burden $<50 \%(n=12)$ and $>50 \%(n=19)$; ET patients were divided into three groups according to mutational status: no mutation $(n=17)$; JAK2V617F allele burden $<50 \%(n=20)$, and $>50 \%(n=12)$. According to treatment, ET patients were divided into two groups: no treatment $(n=7)$ and $\mathrm{HU}$ $(n=41)$. PV patients with JAK2V617 allele burden $>50 \%$ have a lower expression of CD63 after TRAP6 $(25 \mu \mathrm{M})(p=0.04)$, higher percentage of aggregates-PM $(p=0.04)$ and -PMN $(p=0.0014)$, higher expression of CD11b in monocytes $(p=0.015)$ and neutrophils $(p=0.004)$, and of monocyte TF $(p=0.006)$ comparing to those with JAK2V617F allele burden $<50 \%$ (Fig. 2). ET patients with allele burden $>50 \%$ have a statistically significant increase in monocyte CD11b expression comparing to ET no mutation group $(p=0.002)$ and allele burden $<50 \%$ ( $p=0.01$ ) (Fig. 2). Between ET groups, no differences were found regarding PLA and CD11b PMN expression. For all the parameters studied, we did not found statistically significant differences between ET no mutation and ET allele burden $<50 \%$ (Fig. 2). Concerning to cytoreductive treatment no statistically significant differences were found in patients HU-treated or non-HU treated (Fig. 3). 
Fig. 1 Activation parameters in control subjects and in PV and ET groups. Evaluation by flow cytometry of platelet-leukocyte aggregates, leukocyte CD11b, and monocyte tissue factor expression. a Percentage of circulating platelet-monocyte aggregates, ${ }^{\star}{ }^{\S} p<0.0001$ versus CT; $\mathbf{b}$ percentage of circulating platelet-PMN aggregates, ${ }^{*} p=0.01$ and ${ }^{\S} p<0.0001$ versus CT, ${ }^{\phi} p=0.01$ versus ET; c monocyte CD11b expression, ${ }^{*} p=0.0009$ and ${ }^{\S_{p}} p=0.006$ versus CT; d PMN CD11b expression, $p=\mathrm{NS}$; e monocyte tissue factor (CD142), $* p<0.0001$ versus $\mathrm{CT},{ }^{\phi} p=0.003$ versus ET
(A) Platelet-Monocyte aggregates $\mathrm{p}<0.0001$

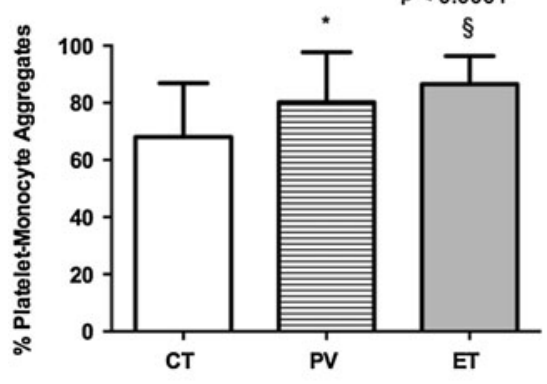

(C) Monocyte CD11b

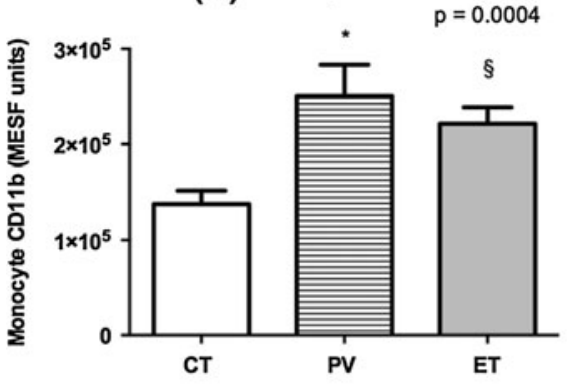

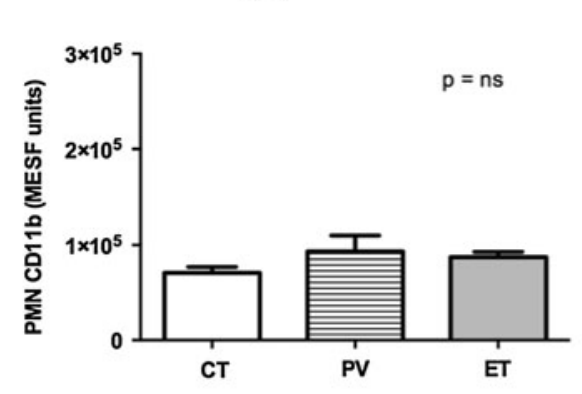

(B) Platelet-PMN aggregates

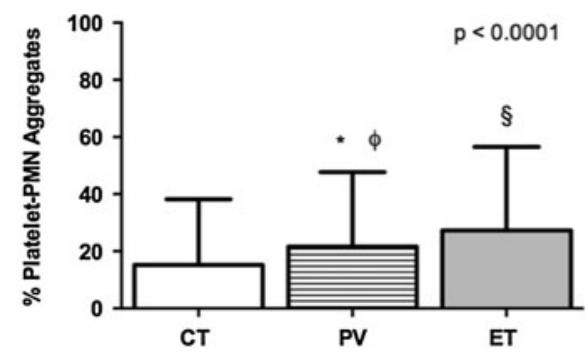

(D) PMN CD11b

(E) Monocyte Tissue Factor (CD142)

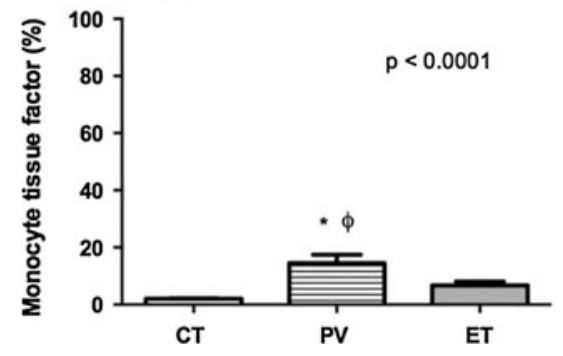

Thrombosis and hemorrhage

In our cohort of ET, the occurrence of thrombosis at diagnosis is more frequent in patients with JAK2V617F mutation $\left(p=0.02, \chi^{2}=5.2\right)$, and in patients with allele burden $>50 \% \quad\left(p=0.02, \chi^{2}=8.3\right)$. ET patients with thrombosis have a statistical significant increase of platelet-neutrophil aggregates (41.2 vs. $23.1, p=0.02)$ and monocyte CD11b expression $\left(272.4 \times 10^{3} \quad\right.$ vs. $150.8 \times 10^{3}$ MESF units, $\left.p=0.02\right)$ and present higher median JAK2V617F allele burden (52.1 vs. $29.6 \%$, $p=\mathrm{NS}$ ) comparing to those without thrombosis. Among PV patients, no statistically significant associations were found regarding thrombosis. In ET patients, hemorrhage was statistically associated with JAK2V617F mutation ( $p=0.01, \chi^{2}=6.7$ ), occurring only in patients with JAK2V617F mutation. In both PV and ET groups, hemorrhagic episodes occurred more frequently in patients with lower median allele burden; however, the differences were not statistically significant.

\section{Discussion}

This study evaluates a group of 80 subjects with MPN, 31 PV, and 49 with ET. All the patients were clinically stable with follow-up periods of 7 years for PV and 8 years for ET; among them, $12 \mathrm{PV}$ and 16 ET patients have a history of thrombosis and $7 \mathrm{PV}$ and 10 ET presented minor hemorrhages. The aims of this study were to investigate whether the presence of JAK2V617F mutation and/or allele burden influence the expression of cellular markers of activation involved in procoagulant activity.

The acquired JAK2V617F mutation results in a gain-offunction of JAK2 that autonomously activates downstream signaling pathways, including JAK-STAT, PI3K/Akt, and ERK1/2 MAPK [16]. In most PV and in a minority of ET patients only the mutated JAK2V617F allele is found in DNA from hematopoietic cells, consistent with loss of heterozygosity for JAK2 locus due to a process of mitotic recombination. This is expected to result in a higher level of JAK/STAT activation, possibly due to loss of 
Fig. 2 Activation parameters in PV and ET patients' according to JAK2V617F allele burden. Patients were divided into three groups: no mutation, allele burden $<50 \%$ and allele burden $>50 \%$. a Platelet-monocyte aggregates, ${ }^{*} p=0.04$ versus $\mathrm{PV}<50 \%$; b platelet-PMN aggregates, $* p=0.0014$ versus $\mathrm{PV}<50 \%$; c monocyte CD $11 \mathrm{~b}$ expression, $* p=0.015$ versus $\mathrm{PV}<50 \%,{ }^{8} p=0.0005$ versus $\mathrm{ET}<50 \%,{ }^{\phi} p=0.04$ versus ET no mut; d PMN CD11b expression, ${ }^{*} p<0.004$ versus $\mathrm{PV}<50 \%$; e monocyte tissue factor $(\mathrm{CD} 142) * p=0.006$ versus $\mathrm{PV}<50 \%$
(A) Platelet-Monocyte aggregates

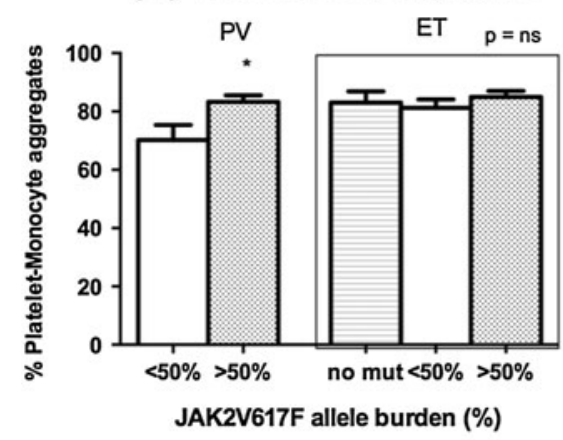

(C) Monocyte CD11b expression

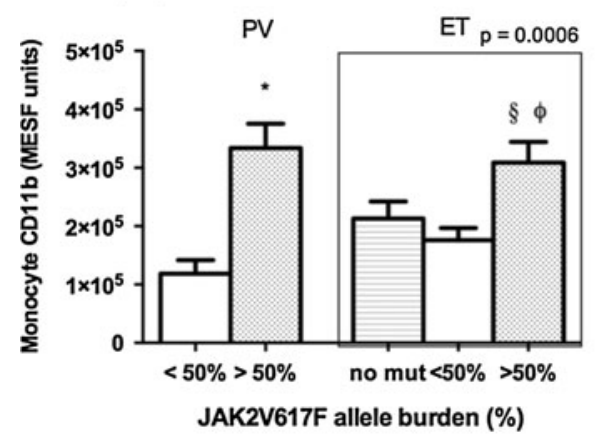

(E) Monocyte Tissue Factor (CD142)

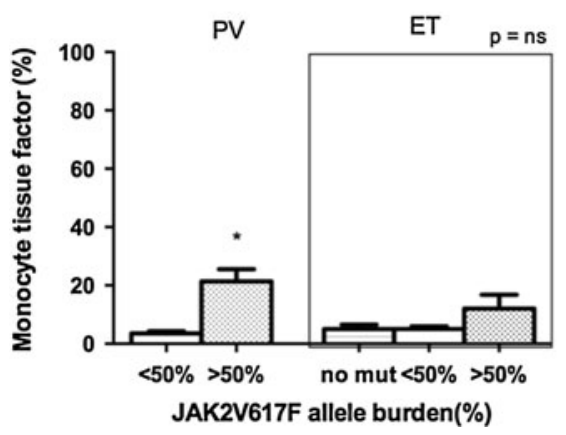

(B) Platelet-PMN aggregates

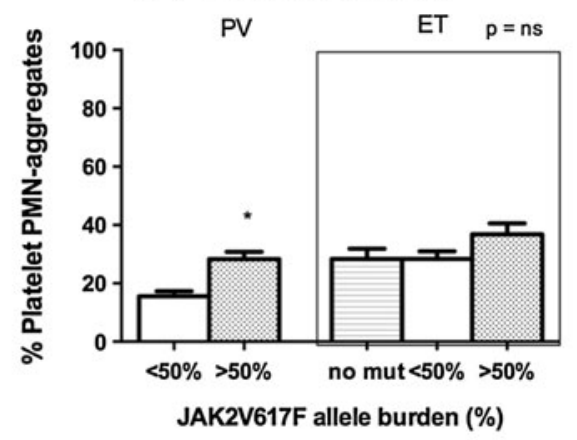

(D) PMN CD11b expression

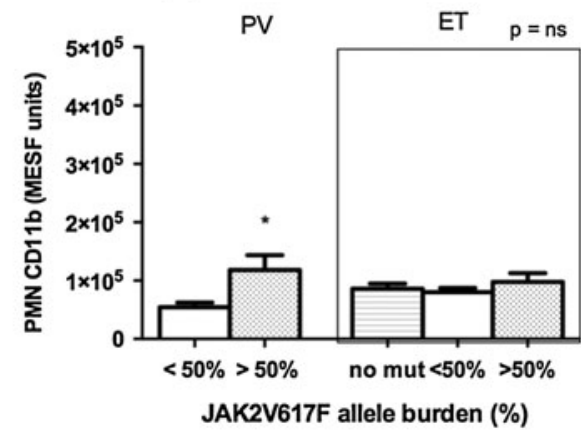

competition of the will type allele [17]. In our study, $61 \%$ of the PV and $38 \%$ of the ET patients have JAK2V617F allele burden $>50 \%$. The mutant allele burden was statistically significantly higher in PV when compared to ET patients (PV $63 \%$; ET $31 \%$ ) and in agreement to the gene dosage hypothesis which suggests that homozygosity favors the erythroid phenotype $[17,18]$. In our cohort, the percentage of JAK2V617F homozygous patients is slightly higher than reported in other studies [19, 20], probably due to the fact that the majority of determinations were performed in follow-up. Other authors found an association between JAK2V617F mutation and thrombotic or hemorrhagic complications in MPN patients [16-18, 20, 21, 22]. Our data shows that in ET patients with JAK2V617F mutation have a statistically significant occurrence of thrombotic events at diagnose and that thrombosis is more frequent in ET patients with allele burden $>50 \%$. We found no association regarding allele burden and thrombosis in PV patients in line with previous reports [20]. Cytoreductive treatment with $\mathrm{HU}$ has been implied to have antithrombotic effect; however, we did not find statistically significant differences in platelet and leukocyte activation markers between ET patients HU-treated and non-HUtreated. These data suggest that $\mathrm{HU}$ has no effect in decreasing the activation status, and the antithrombotic effect may be mainly due to decrease in leukocytes number, which has been considered a major thrombotic risk [6]. To investigate if JAK2V617F allele burden influenced platelet and leukocyte activation, we tested different cellular activation markers and found an increased membrane expression of both CD62P (P-selectin) and CD63 (granulophysin) in non-stimulated platelets, indicating platelet activation. Similar observations were reported by other groups $[8,11,23]$. We observed a statistically significant 
Fig. 3 Comparison of activation parameters in ET patients regarding hydroxyurea treatment. No statistically significant differences were observed comparing ET patients non-treated (non-HU) and treated with hydroxyurea (HU). a Platelet-leukocyte aggregates; b leukocyte CD11b expression; c monocyte tissue factor expression

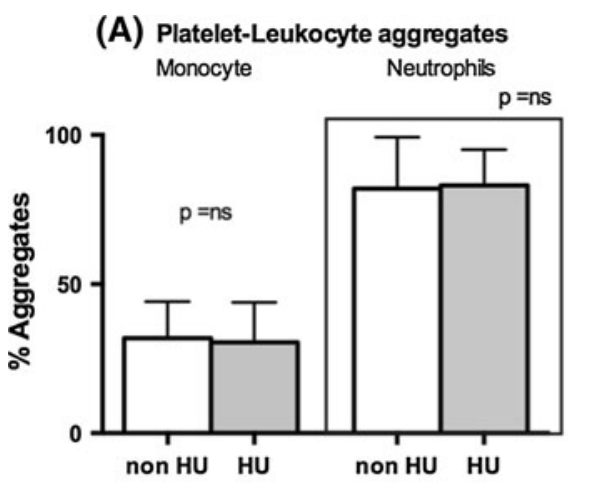

(B) Leukocyte CD11b expression

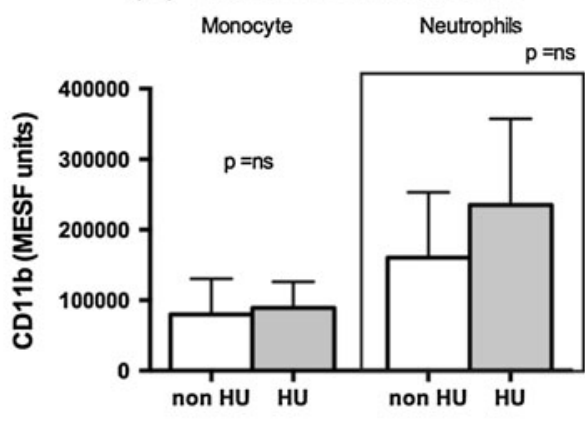

(C) Monocyte Tissue Factor (CD142)

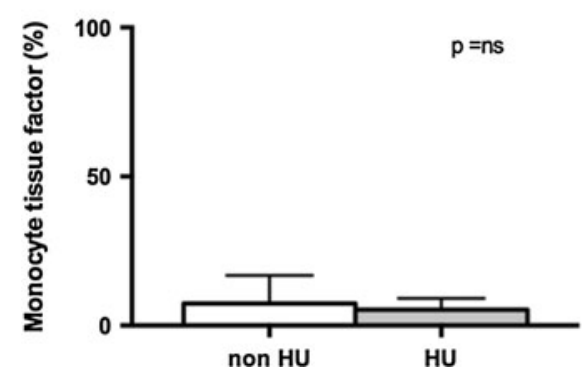

increase response to arachidonic acid and diminished expression of CD62P and CD63 following TRAP6 activation, comparing to controls. The increased response to arachidonic acid could be explained by an increased and sustained thromboxane A2 (TXA2) generation, which has been reported in $40 \%$ of MPN patients, in correlation with lipoxygenase deficiency [4]. As it has been suggested, we observed an acquired storage pool disease in $71 \% \mathrm{PV}$ and in $47 \%$ ET patients that, in these patients, might be due to continuous activation, conjugated with abnormal receptor mediated granule secretion consistent with a low response to TRAP6 agonist. The adhesion of activated platelets to monocytes and PMN is mediated through binding of platelet P-selectin to P-selectin glycoprotein ligand-1 (PSGL-1) expressed in leukocytes, generating mixed aggregates, with subsequent activation of $\beta 2$ integrin CD11b/CD18 [24]. Increased circulating PLA have been previously demonstrated in several pathological conditions to be associated with thrombosis propensity [10, 24]. Accordingly, when comparing to controls, we found a statistically significant increase of circulating PMs and platelet-PMN aggregates in PV and ET patients, confirming previous findings $[12,25]$. ET patients, platelet-PMN aggregates were higher than in PV, with a positive correlation found between platelet number and PLA, evidencing that thrombocytosis favors the aggregates formation. However, circulating PLA may be triggered not only by P-selectin expression at platelets surface, but also by activated leukocytes [25]. Whether the presence of activated leukocytes promotes platelets activation and how this interaction can lead to hemostatic activation is unclear. During cellular activation, leukocytes undergo phenotypic modifications with changes in adhesion molecules expression at the cellular surface [25]. The CD11b integrin in leukocytes is currently accepted as a marker of activation and is responsible for the firm attachment of leukocytes to endothelium and platelets. Furthermore, the cooperation between activated leukocytes and platelets is suggested to be involved in TF generation and activation of extrinsic coagulation system (TF binding to factor VII/VIIa) [24]. TF expression is up-regulated by a number of pathophysiological agonists, as well as following P-selectin binding to PSGL-1 on monocytes. We demonstrate that ET and PV patients have activated monocytes and PMNs leukocytes in circulation, as they have statistically significant increase in $\mathrm{CD} 11 \mathrm{~b}$ and monocyte-TF when comparing to control subjects, in line with other authors $[8,11]$. We also found that TF expression is higher in PV than in ET patients, with statistically significant differences, and is correlated with monocyte $\mathrm{CD} 11 \mathrm{~b}$ expression, suggesting that monocyte $\mathrm{TF}$ expression is related to monocyte activation. Markers of platelet-leukocyte activation have been shown to be higher in ET patients with a history of thrombosis compared to patients without thrombosis $[12,23]$. In agreement with previous studies, we found, with statistical significance, increased levels of platelet-neutrophil aggregates and higher expression of monocyte CD11b in ET patients with thrombosis compared to ET without thrombosis [12, 23]. From our observations of a consistent activation of platelets and leukocytes in PV and ET patients, is expected to 
have a correlation with the intensity of constitutive JAK2/ STAT pathway activation. To access this hypothesis, we studied the influence of allele burden in platelet and leukocyte activation. Regarding platelet studies in PV and ET patients, we found an inverse statistically significant correlation between JAK2V617F allele burden and CD63 expression after TRAP6 $(25 \mu \mathrm{M})$, which is accordance with the high prevalence of storage pool phenotype in both group of patients. Considering data altogether, platelet activation and response to agonists are not directly correlated with allele burden. Concerning leukocyte activation, we found statistically significant correlations between JAK2V617F allele burden and the amount of platelet-neutrophil aggregates, PMN and monocyte CD11b expression, and TF expression in PV patients; and a statistically significant correlation between JAK2V617F allele burden and monocyte CD11b expression in ET patients. Also, comparing the ET groups without mutation with controls we did not find significant differences in monocyte and PMN CD11b expression, and, although no significantly differences, PV patients have the highest levels of CD11b both in monocytes and PMN. These data strongly suggest that JAK2V617F allele burden may have a direct influence in leukocyte activation, and this influence is more obvious in the PV patients group, most likely because of the higher levels of the mutant JAK2V617F allele.

In conclusion, this study provides important evidences regarding the association of JAK2V617F allele burden with the prothrombotic status in MPN patients. Increased levels of PLA, CD11b, and TF expression were directly associated with JAK2V617F allele burden. In ET, increased levels of platelet-neutrophil aggregates and monocyte CD11b expression were associated with thrombosis, supporting the role of leukocyte activation in the pathophysiology of thrombosis. Activation parameters studied here were not susceptible to HU treatment; therefore, the new JAK2 inhibitors may have an important role preventing leukocyte activation, thus decreasing the thrombotic risk in these patients.

Acknowledgments This study was supported by the Grant PTDC/ SAU-GMG/74375/2006 from the Portuguese Foundation for Science and Technology, FCT, Portugal.

Conflict of interest The authors declare no competing financial interests.

\section{References}

1. Landolfi R, Cipriani MC, Novarese L. Thrombosis and bleeding in polycythemia vera and essential thrombocythemia: pathogenetic mechanisms and prevention. Best Pract Res Clin Hematol. 2006;19:617-33.

2. Elliott MA, Tefferi A. Thrombosis and hemorrhage in polycythemia vera and essential thrombocythemia. $\mathrm{Br} \mathrm{J}$ Haematol. 2004;128:275-90.
3. Harrison CN. Platelets and thrombosis in myeloproliferative diseases. Hematology Am Soc Hematol Educ Program. 2005;409-15.

4. Schafer AI. Bleeding and thrombosis in the myeloproliferative disorders. Blood. 1984;64:1-12.

5. Landolfi R, Gennaro LD, Falanga A. Thrombosis in myeloproliferative disorders: pathogenetic facts and speculation. Leukemia. 2008;22:2020-8.

6. Landolfi R, Gennaro L, Barbui T, et al. Leukocytosis as a major thrombotic risk factor in patients with polycythemia. Blood. 2007;109:2446-52.

7. Fallanga A, Marchetti M, Evangelista V, et al. Polymorphonuclear leukocyte activation and hemostasis in patients with essential thrombocythemia and polycythemia vera. Blood. 2000;96:4261-6.

8. Falanga A, Marchetti M, Vignoli A, Balducci D, Barbui T. Leukocyte-platelet interaction in patients with essential thrombocythemia and polycythemia vera. Exp Hematol. 2005;33:523-30.

9. Passamonti F, Rumi E, Pietra D, et al. Relation between JAK2(V617F) mutation status, granulocyte activation and constitutive mobilization of CD34-positive cells into peripheral blood in myeloproliferative disorders. Blood. 2006;107:676-3682.

10. Jensen MK, de Nully Brown P, Lund BV, Nielsen OJ, Hasselbalch HC. Increased circulating platelet-leukocyte aggregates in myeloproliferative disorders is correlated to previous thrombosis, platelet activation and platelet count. Eur J Haematol. 2001;66:143-51.

11. Rodrigo EA, Alvarez-Larrán A, Reverter JC, Villamor N, Colomer D, Cervantes F. Increased platelet and leukocyte activation as contributing mechanisms for thrombosis in essential thrombocythemia and correlation with the JAK2 mutational status. Haematologica. 2006;91:169-75.

12. Falanga A, Marchetti M, Vignoli A, et al. V617F JAK-2 mutation in patients with essential thrombocythemia: relation to platelet, granulocyte, and plasma hemostatic and inflammatory molecules. Exp Hematol. 2007;35:702-11.

13. Austin SK, Lambert R. The JAK2V617F mutation and thrombosis. Br J Haematol. 2008;143:307-20.

14. Vannucchi AM, Antonioli E, Guglielmelli P, Pardanani A, Tefferi A. Clinical correlates of JAK2V617F presence or allele burden in myeloproliferative neoplasms: a critical reappraisal. Leukemia. 2008;22:1299-307.

15. Chen Q, Lu P, Jones AV, Cross NC, Silver RT, Wang YL. Amplification refractory mutation system, a highly sensitive and simple polymerase chain reaction assay, for the detection of JAK2V617F mutation in chronic myeloproliferative disorders. J Mol Diagn. 2007;9:272-6.

16. Vannucchi AM. Insights into the pathogenesis and management of thrombosis in polycythemia vera and essential thrombocythemia. Intern Emerg Med. 2010;5:177-84.

17. Vannucchi AM, Guglielmell P. Molecular pathophysiology of Philadelphia-negative myeloproliferative disorders: beyond JAK2 and MPL mutations. Haematologica. 2008;93:972-6.

18. Passamonti F, Rumi E. Clinical relevance of JAK2(V617F) mutant allele burden. Haematologica. 2009;94:7-10.

19. Antonioloi E, Guglielmelli P, Poli G, et al. Influence of JAK2V617F allele burden on phenotype in essential thrombocythemia. Haematologica. 2008;93:41-7.

20. Passamonti F, Rumi E, Pietra D, et al. A prospective study of 388 patients with polycythemia vera: impact of JAK2(V617F) allele burden and leukocytosis on fibrotic or leukemic disease transformation and vascular complications. Leukemia. 2010;24:1574-9.

21. Carobbio A, Finazzi G, Antoniolo E, et al. JAK2V617F allele burden and thrombosis: a direct comparison in essential thrombocythemia and polycythemia vera. Exp Hematol. 2009;37: 1016-21. 
22. Carobbio A, Thiele J, Passamonti F, et al. Risk factors for arterial and venous thrombosis in WHO-defined essential thrombocythemia: an international study of 891 patients. Blood. 2011;117: 5857-9.

23. Jensen MK, Brown PN, Lund BV, Nielsen OJ, Hasselbalch HC. Increased platelet activation and abnormal membrane glycoprotein content and redistribution in myeloproliferative disorders. $\mathrm{Br}$ J Haematol. 2000;110:116-24.
24. McEver RP. P-selectin/PSGL-1 and other interactions between platelets, leukocytes and endothelium. In: Platelets. Chapter 12, 2nd ed. Amsterdam: Elsevier; 2007. p. 231-42.

25. Falanga A, Marchetti M, Barbui T, Smith W. Pathogenesis of thrombosis in essential thrombocythemia and polycythemia vera: the role of neutrophils. Semin Hematol. 2005;42:239-47. 